\title{
Efecto del glicerol sobre la catálisis por fosfatasa ácida de bajo peso molecular de hígado de alpaca (Lama pacos)
}

\author{
Emilio Guija ${ }^{1}$, Fernando Arauco ${ }^{2}$, Hielke Haak-Mares ${ }^{\dagger}$, Mercedes Soberón ${ }^{1}$
}

Resumen

Palabras clave

\begin{abstract}
Objetivo: Determinar el efecto del glicerol sobre la hidrólisis del p-nitrofenil fosfato a pH 5,0 por fosfatasa ácida de bajo peso molecular de hígado de alpaca. Diseño: Estudio analítico experimental. Lugar: Centro de Investigación de Bioquímica y Nutrición, Facultad de Medicina, Universidad Nacional Mayor de San Marcos. Materiales: Se utilizó los reactivos químicos p-nitrofenil fosfato sal disódica, ácido acético glacial, glicerol, ácido tricloroacético, ácido sulfúrico, molibdato de amonio, ácido ascórbico, sulfato de amonio, sephadex G-75 (45-120), sulfoetil sephadex C-50 y etilendiaminotetraacético (EDTA). Métodos: Se determinó los parámetros cinéticos Km y Vmax utilizando como sustrato el p-nitrofenil fosfato, en presencia de concentraciones variables de glicerol. Así mismo, se determinó la velocidad de liberación de los productos de la reacción en función de la concentración de dicho nucleófilo. Principales medidas de resultados: Efecto del glicerol sobre la hidrólisis del p-nitrofenil fosfato. Resultados: El glicerol en concentraciones comprendidas entre 1,16 y 3,49 M incrementó linealmente la liberación del p-nitrofenol; en cambio, la formación del fosfato inorgánico -el segundo producto liberado- no se modificó. Así mismo, los valores de Km y Vmax se incrementaron linealmente dependientes de las concentraciones de glicerol, en un rango comprendido entre 0,58 y 2,32 M. Conclusiones: Un análisis de las modificaciones que ejerce el glicerol sobre los valores de Km y Vmax y la velocidad de liberación de los productos de la reacción permite sugerir un modelo en el que la fosfatasa ácida de bajo peso molecular de hígado de alpaca cataliza la hidrólisis de fosfomonoésteres a través de un mecanismo uni biordenado, con la formación de un complejo enzima-fosfato, que sería escindido por agua o un nucleófilo, como el glicerol; en este modelo a $k_{2}$ le corresponde un valor mucho mayor que $k_{3} y k_{4} N$.

Fosfatasa ácida; catálisis; glicerol; cinética.
\end{abstract}

Effect of glycerol on catalysis by low molecular weight acid phosphatase from alpaca liver (Lama pacos)

Abstract

Objective: To determine the effect of glycerol on hydrolisis of p-nitrophenyl phosphate at $\mathrm{pH}$ 5,0 by low molecular weight acid phosphatase from alpaca liver. Design: Experimental analytical study. Setting: Biochemistry and Nutrition Research Center, Faculty of Medicine, Universidad Nacional Mayor de San Marcos, Lima, Peru.

1 Profesor Emérito del Centro de Investigación de Bioquímica y Nutrición. Facultad de Medicina, Universidad Nacional Mayor de San Marcos. Lima, Perú.

2 Unidad de Post Grado. Facultad de Medicina, Universidad Nacional Mayor de San Marcos. Lima, Perú.

+ El presente artículo se publica como un homenaje póstumo al Dr. Hielke Haak Mares, Profesor Principal de la Facultad de Medicina de la Universidad Nacional Mayor de San Marcos. Lima, Perú.
Materials: Disodic p-nitrophenyl phosphate salt, glacial acetic acid, glycerol, tricloroacetic acid, sulfuric acid, ammonium molibdate, ascorbic acid, ammonium sulphate, sephadex G-75 (45-120), sulpho ethyl sephadex C-50 and ethylene diaminotetraacetic (EDTA) chemical reactives. Methods: Both Km and Vmax kinetic parameters were determined with p-nitrophenyl phosphate as substrate in presence of different concentrations of glycerol. The rate of formation of products was determined as a function of the concentration of such nucleophile. Main outcome measures: Glycerol effect on p-nitrophenyl phosphate hydrolysis. Results: Glycerol linearly increased pnitrophenol release at concentrations between 1,16 and 3,49M. Instead, inorganic phosphate formation, the second product, was not modified. Also, Km and Vmax values increased linearly between 0,58 and 2,32 $M$ depending on glycerol concentrations. Conclusions: Analysis of modifications induced by glycerol on $\mathrm{Km}$ and Vmax 
values as well as on liberation velocity of the reaction products suggests a model in which low molecular weight acid phosphatase isolated from alpaca liver catalyses phosphomonoesters hydrolysis through an uni biordered mechanism, with formation of an enzyme-phosphate complex that will be splitted by water or a nucleophile such a glycerol; in this model, $k_{2}$ corresponds to a much higher value than $k_{3}$ or $k_{4} N$.

Key words: Acid phosphatase; catalysis; glycerol; kinetics.

\section{INTRODUCCIÓN}

La fosfatasa ácida (EC 3.1.3.2) es una enzima de amplia distribución en la naturaleza. Se ha descrito su purificación a partir de diversas fuentes: hígado $\left({ }^{1}\right)$, próstata $\left({ }^{2}\right)$, cerebro $\left({ }^{3}\right)$, germen de trigo $\left.{ }^{4}\right)$, papa $\left(^{5}\right)$, cerdo $\left(^{6}\right)$, etc. Existen fosfatasas ácidas de diverso peso molecular, caracterizándose las de bajo peso molecular por no requerir de cofactor para ejercer su acción catalítica, hecho que permite simplificar considerablemente la interpretación de los resultados experimentales $\left(^{7}\right)$.

Un aumento en los niveles de fosfatasa ácida sérica está relacionado con carcinoma de próstata, infarto prostático, traumatismo prostático, hipertrofia benigna de próstata, prostatitis, destrucción excesiva de plaquetas (púrpura trombocitopénica idiopática, con megacariocitos en médula ósea), crisis hemolíticas, tromboembolismo, carcinoma metastático del hueso, enfermedad de Paget ósea avanzada, hepatitis, ictericia obstructiva, cirrosis hepática, hiperparatiroidismo, alteración renal aguda, entre otros. La fosfatasa ácida prostática (PAP) es un subtipo de la fosfatasa ácida sérica o total. Existen cuatro formas de isoenzimas de fosfatasa ácida a nivel estructural de genes $\left({ }^{8,9}\right)$. Las formas eritrocíticas y lisosomales están expresadas en la mayoría de las células, mientras que, la prostática y la forma macrofágica tienen una expresión limitada. La forma prostática (PAP) es específica para neoplasias de la glándula prostática, siendo el principal marcador bioquímico del cáncer de próstata hasta la aparición del antígeno prostático específico (PSA) $\left({ }^{10,11}\right)$.

Esta enzima cataliza la hidrólisis de los fosfomonoésteres, como el p-nitrofenilfosfato, a través de un proceso cinético uni-biordenado, caracterizado por liberarse en primer lugar el p-nitrofenol y ulteriormente lo haría el fosfato inorgánico $\left({ }^{12}\right)$. En esta secuencia de reacción se formaría un complejo intermedio covalente enzima-fosfato, el que probablemente sería tasa limitante de la reacción total del proceso catalítico $\left({ }^{12}\right)$. Se ha observado en fosfatasas ácidas de otras fuentes que el fosfato se ligaría a un residuo de histidina $\left({ }^{4}\right)$, aunque no se descarta la posibilidad que dicha interacción podría realizarse con la participación de otros residuos de aminoácidos $\left({ }^{13}\right)$.

La utilización de otros nucleófilos más eficientes que el agua ha sido usada con la finalidad de describir de una manera más precisa el mecanismo de acción de diversas hidrolasas. El presente trabajo muestra el efecto del glicerol sobre la hidrólisis del p-nitrofenil fosfato a $\mathrm{pH} 5,0$ por fosfatasa ácida de bajo peso molecular de hígado de alpaca.

\section{MÉTODOS}

Se realizó un estudio de tipo analítico experimental, utilizándose los siguientes reactivos químicos: p-nitrofenil fosfato sal disódica, ácido acético glacial, glicerol, ácido tricloroacético, ácido sulfúrico, molibdato de amonio, ácido ascórbico, sulfato de amonio, sephadex G-75 (45-120), sulfoetil sephadex C-50 y etilendiaminotetraacético (EDTA).

La purificación de la enzima se realizó conforme se ha descrito previamente, con algunas modificaciones realizadas por el Dr. Hielke Haak $\left({ }^{14}\right)$. La actividad de la fosfatasa ácida se determinó en un medio de ensayo que estuvo constituido en un volumen final de 2,0 mL por tampón acetato $0,05 \mathrm{M}$ pH 5,0, EDTA $1 \times 10^{-4} \mathrm{M}, 2 \mu$ moles de p-nitrofenil fosfato y enzima. Después de una incubación de 2 minutos a $37^{\circ} \mathrm{C}$, la reacción se detuvo mediante la adición de $1 \mathrm{~mL}$ de $\mathrm{NaOH} 1 \mathrm{~N}$. El p-nitrofenolato liberado se midió a $400 \mathrm{~nm}$ en un espectrofotómetro Gilford modelo 240, habiéndose utilizado el blanco respectivo que no contenía enzima. Para realizar los cálculos se utilizó un coeficiente de extinción molar de $1,8 \times 10^{4} \mathrm{M}^{-1} \mathrm{~cm}^{-1}$. La liberación del p- 
nitrofenol bajo estas condiciones no excedió el 3\% del sustrato utilizado $\left({ }^{15}\right)$.

Para la determinación del fosfato inorgánico liberado durante la catálisis por fosfatasa ácida, se utilizó el medio anteriormente descrito, pero la reacción se detuvo a los 2 minutos por adición de $1 \mathrm{~mL}$ de ácido tricloroacético al 10\%. Luego, se tomó una alícuota de $1 \mathrm{~mL}$, se le añadió $3 \mathrm{~mL}$ de agua destilada y $4 \mathrm{~mL}$ del reactivo de color; se incubó a $37^{\circ} \mathrm{C}$ durante 90 minutos, a cuyo término se leyó la absorbancia a $820 \mathrm{~nm}$. Para cada determinación se preparó el blanco respectivo que no contenía enzima. Con el propósito de realizar los cálculos, se preparó una curva patrón, utilizando fosfato inorgánico en un rango comprendido entre 0,5 y $8,0 \mu \mathrm{g}\left({ }^{16}\right)$.

Para las determinaciones de los valores de $\mathrm{Km}$ y Vmax se utilizó como sustrato el p-nitrofenilfosfato, en concentraciones comprendidas entre $5,0 \mathrm{x}$ $10^{-5} \mathrm{M}$ y $1,0 \times 10^{-3} \mathrm{M}$. Se realizó los cálculos de los parámetros antes mencionados usando el método de Lineweaver y Burk $\left({ }^{17}\right)$, para cuya finalidad se graficó en doble recíproca las inversas de las

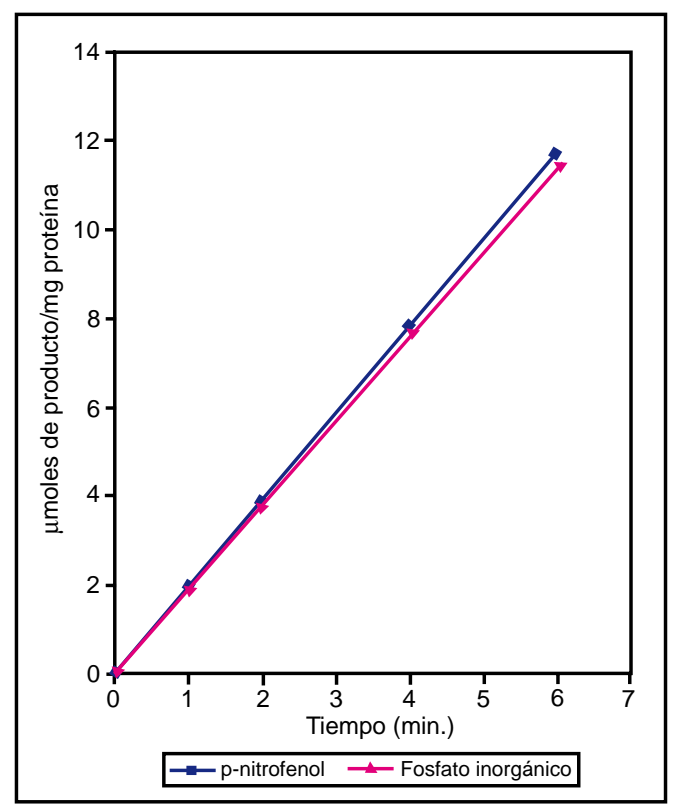

Figura 1. Hidrólisis enzimática del p-nitrofenil fosfato. Liberación de p-nitrofenol y fosfato inorgánico en función del tiempo. velocidades de reacción en función de las inversas de las concentraciones de sustrato.

\section{RESULTADOS}

La fosfatasa ácida de bajo peso molecular de hígado de alpaca hidrolizó el p-nitrofenil fosfato a $\mathrm{pH} 5,0$ y $37^{\circ} \mathrm{C}$, liberando en el curso de la reacción los productos p-nitrofenol y fosfato inorgánico, en una relación molar de $1: 1$, durante un periodo de seis minutos, conforme se muestra en el Figura 1. La adición de glicerol a una concentración 1,16 $\mathrm{M}$ incrementó considerablemente la velocidad de reacción, en aproximadamente el doble de aquella observada en ausencia del nucleófilo, lo que al mismo tiempo modificó la relación estequiométrica del p-nitrofenol con respecto al fosfato inorgánico liberado, cuyo valor en el curso de la reacción fue de 1,67, resultado que se observa en la Figura 2.

En la Figura 3 se aprecia las modificaciones de la velocidad de formación del p-nitrofenol y del fosfato inorgánico en función de las concentraciones de glicerol; en dicho gráfico se observa

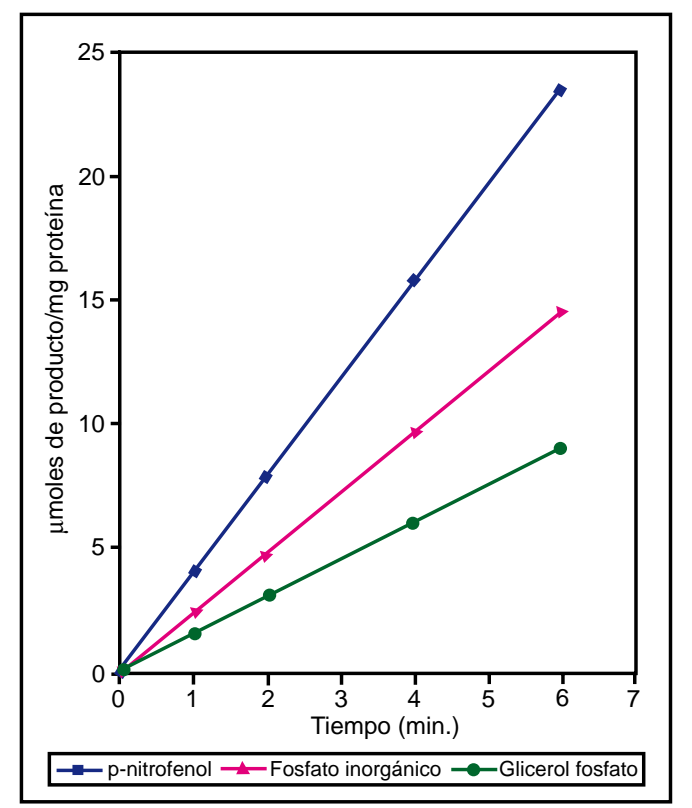

Figura 2. Hidrólisis enzimática del p-nitrofenil fosfato en presencia de glicerol Efecto del tiempo sobre la liberación de productos. 


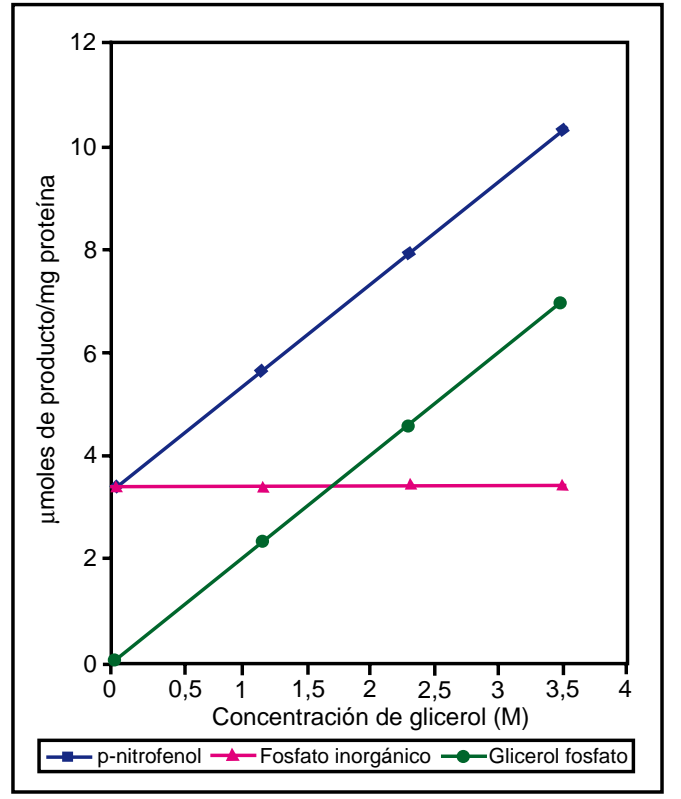

Figura 3. Efecto del glicerol sobre la velocidad de hidrólisis del p-nitrofenil fosfato. Liberación de productos.

que la velocidad de liberación del p-nitrofenol varía linealmente a medida que se incrementa la concentración de glicerol. En cambio, la liberación del fosfato inorgánico no se modifica. Estos resultados sugieren que la formación del glicerol-

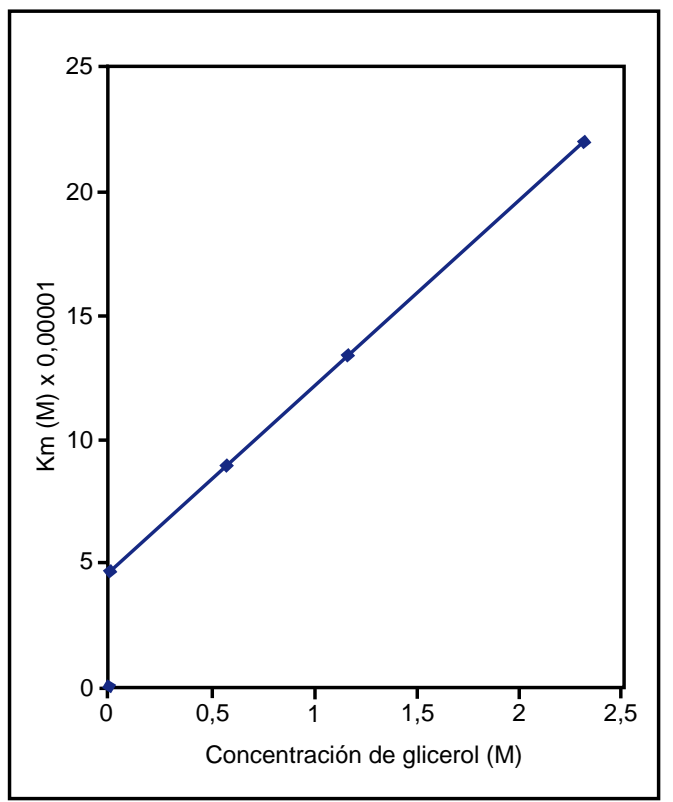

Figura 4. Efecto del glicerol sobre el valor Km. fosfato durante el proceso de catálisis se liberaría linealmente dependiente de la concentración del glicerol adicionado al medio de ensayo.

El efecto del glicerol sobre los parámetros cinéticos de $\mathrm{Km}$ y Vmax se realizó utilizando tres concentraciones diferentes de glicerol, en un rango comprendido entre $0,58 \mathrm{M}$ y $2,32 \mathrm{M}$. En la Figura 4 puede observarse que el valor $\mathrm{Km}$ varía linealmente en función de las concentraciones de glicerol utilizadas. Un efecto similar se observa con el incremento de la velocidad máxima, conforme se muestra en la Figura 5.

\section{DISCUSIÓN}

Se ha mostrado que la fosfatasa ácida prostática (PAP) puede ser un marcador muy útil en el tamizaje y evaluación de la enfermedad activa y respuesta al tratamiento del linfoma intravascular de las células $\mathrm{B}$, que muchas veces es tratado como fiebre de origen desconocido $\left({ }^{11,18}\right)$.

Las fosfatasas ácidas son enzimas que tienen la propiedad de hidrolizar ésteres monofosfóricos, a un $\mathrm{pH}$ de 5,0, liberando como productos de la reacción un alcohol, cuya naturaleza depende del

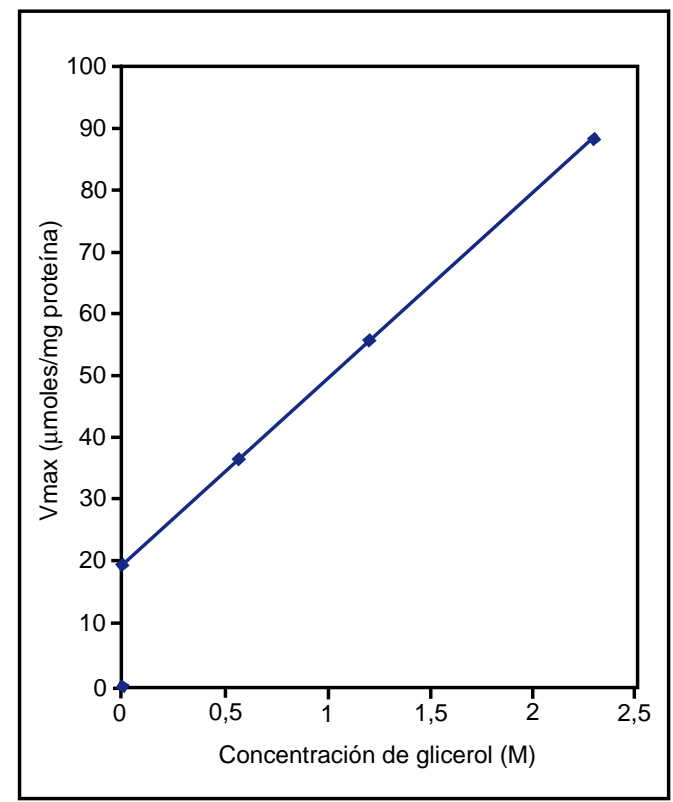

Figura 5. Efecto del glicerol sobre la velocidad máxima. 
sustrato utilizado, y fosfato inorgánico $\left({ }^{7,19,20}\right)$. Se ha sugerido que la enzima hidrolizaría los sustratos a través de un proceso de catálisis covalente, con la probable formación de un complejo intermedio enzima-fosfato, el que posteriormente se hidrolizaría liberando el fosfato inorgánico $\left({ }^{12}\right)$. Este proceso mecanístico podría comprender la formación de otros complejos que se formarían en una etapa previa a la liberación del fosfato inorgánico, por cuyo motivo, el uso de reactivos nucleófilos más eficaces que el agua brindaría nuevas posibilidades mecanísticas a considerar, para explicar de una manera más apropiada el proceso de catálisis. Diversos autores han sugerido el modelo uni biordenado, utilizando la nomenclatura propuesta por Cleland $\left({ }^{21}\right)$, para explicar la catálisis por fosfatasa ácida de hígado de bovino $\left({ }^{12}\right)$, hígado de cerdo $\left({ }^{22}\right)$ y cerebro de bovino $\left({ }^{23}\right)$.

La utilización de nucleófilos en reacciones de esta naturaleza permite desarrollar ecuaciones de velocidad para una gran diversidad de modelos mecanísticos. Nuestros resultados podrían explicarse de una manera más apropiada si asumimos que el complejo enzima-fosfato reacciona con el glicerol, previa suposición de que $\mathrm{k}_{2}$ es apreciablemente mayor que $\mathrm{k}_{3}$ y $\mathrm{k}_{4}$ [N], lo que podría ajustarse al siguiente modelo de la Figura 6.

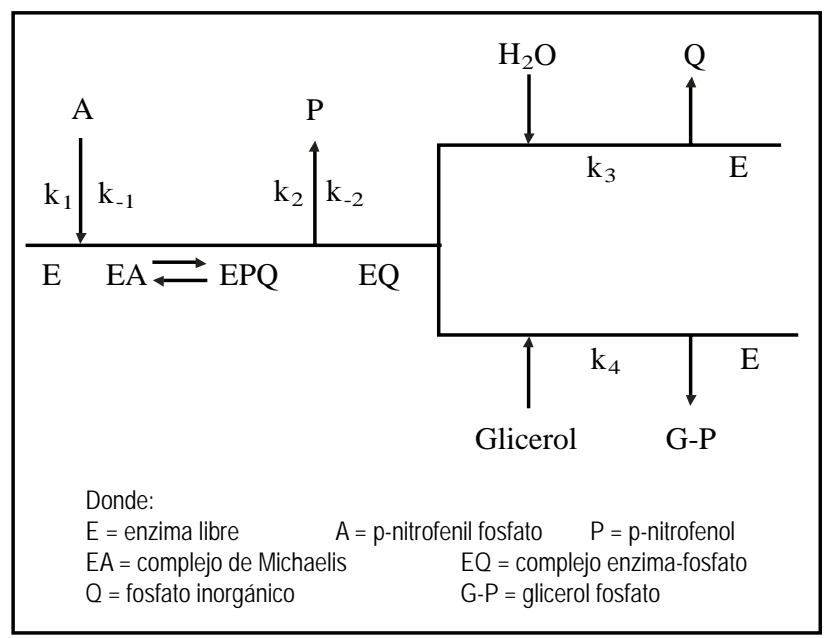

Figura 6. Modelo de la catalisis por fosfatasa ácida de bajo peso molecular en presencia de nucleófilo: glicerol.
En este modelo se considera que el complejo EQ reaccionaría con el agua o con el glicerol y que el valor de $\mathrm{k}_{2}$ sería mayor que $\mathrm{k}_{3}\left[\mathrm{H}_{2} \mathrm{O}\right] \cong \mathrm{k}_{4}$ [glicerol], de tal manera que la liberación de pnitrofenol estaría definido por: $\mathrm{k}_{\mathrm{G}}=\mathrm{k}_{3}\left[\mathrm{H}_{2} \mathrm{O}\right]+$ $\mathrm{k}_{4}$ [licerol].

Conforme se aprecia en la Figura 3, de manera análoga, la liberación de fosfato inorgánico no se modificaría al variar la concentración de glicerol, comportamiento que se ajustaría a la ecuación: $\mathrm{k}_{\mathrm{pi}}$ $=\mathrm{k}_{3}\left[\mathrm{H}_{2} \mathrm{O}\right]$, es decir: $\mathrm{k}_{\mathrm{pi}}=\mathrm{k}_{3}^{\prime}$ Asimismo, la velocidad de liberación del glicerol-fosfato obedece a la ecuación $\mathrm{k}_{\mathrm{G}-\mathrm{P}}=\mathrm{k}_{4}[\mathrm{~N}]$, tal como se observa en la Figura 3. Para el modelo propuesto, la constante de Michaelis-Menten correspondería a:

$$
\mathrm{Km}=\frac{\mathrm{k}_{-1}+\mathrm{k}_{2}}{\mathrm{k}_{1}} \times \frac{\mathrm{k}_{3}+\mathrm{k}_{4}[\mathrm{~N}]}{\mathrm{k}_{2}}
$$

La liberación lineal del p-nitrofenol en función de la concentración de glicerol sugiere un modelo en el que $\mathrm{k}_{2}$ tendría un valor mayor que $\mathrm{k}_{3} \mathrm{y}_{4}[\mathrm{~N}]$ o que los productos fosfato inorgánico, $\mathrm{p}$-nitrofenol y el nucleófilo fosforilado se liberarían a una velocidad mayor o similar a $\mathrm{k}_{-1}$. Pero, este mecanismo podría descartarse si se tiene en consideración que las fosfatasas ácidas catalizan las reacciones a través de un proceso uni-biordenado $\left({ }^{12,22,23}\right)$. Así mismo, un mecanismo en el que $\mathrm{k}_{2}<\mathrm{k}_{3} \mathrm{o}_{2}<$ $\mathrm{k}_{4}[\mathrm{~N}]$ tendría que descartarse, ya que en este tipo de mecanismo la liberación del p-nitrofenol sería independiente de la concentración de glicerol.

El comportamiento de tipo hiperbólico mostrado por la fosfatasa ácida de hígado de bovino para liberar el p-nitrofenol en presencia de diferentes concentraciones de glicerol se explicaría admitiendo que los valores de $\mathrm{k}_{2}, \mathrm{k}_{3} \mathrm{y}_{4}$ [N] fuesen similares $\left({ }^{24}\right)$, por cuyo motivo, dicho modelo no podría explicar los resultados que hemos obtenido en el presente trabajo, los que se ciñen más apropiadamente a un modelo, donde el valor de $\mathrm{k}_{2}$ es mayor que $\mathrm{k}_{3} \mathrm{y} \mathrm{k}_{4}[\mathrm{~N}]$.

Se ha sugerido para la hidrólisis de p-nitrofenil ésteres lipídicos por la lipasa lipoproteica, un mecanismo similar al que proponemos en el presente 
trabajo; en el mencionado proceso se utiliza como nucleófilo a la hidroxilamina, compuesto que incrementa los valores de Km y Vmax, sugiriéndose que el nucleófilo proporcionaría una ruta alterna para la descomposición del intermediario acil-lipasa lipoproteica, el cual constituye la etapa limitante en dicha secuencia catalítica $\left.{ }^{25}\right)$. El incremento lineal de $\mathrm{Km}$ y Vmax en función de la concentración de glicerol, que hemos observado con la fosfatasa ácida de bajo peso molecular de hígado de alpaca, se explica mejor con el modelo cinético que se propone en el presente trabajo.

Así mismo, el incremento de la concentración de glicerol en el medio de reacción no produjo aumento alguno de la liberación de fosfato inorgánico, resultado que se explica de acuerdo al modelo que hemos propuesto, el que predice que la liberación de fosfato inorgánico es independiente de la concentración de glicerol. Este resultado difiere de aquellos obtenidos con la fosfatasa ácida de hígado de bovino $\left({ }^{24}\right)$, en el que la liberación de fosfato inorgánico disminuye a medida que se incrementa la concentración de glicerol, resultado que se corrobora mediante la regraficación en recíproca simple de la liberación de fosfato inorgánico en función de la concentración de glicerol. Análogamente, la liberación del nucleófilo fosforilado en la reacción catalizada por fosfatasa ácida de hígado de bovino es compatible con un mecanismo en el que $\mathrm{k}_{2}$ tiene valores similares a $\mathrm{k}_{3} \mathrm{y} \mathrm{k}_{4}[\mathrm{~N}]$. En cambio, los resultados del presente trabajo se ajustan más adecuadamente a la liberación de glicerol-fosfato, directamente dependiente de la concentración de glicerol. Es necesaria la utilización de otros sustratos en experimentos de transfosforilación para explicar de una manera más precisa la naturaleza de los complejos intermedios que se formarían en la secuencia de reacciones catalizadas por fosfatasa ácida.

\section{REFERENCIAS BIBLIOGRÁFICAS}

1. Taga E, Van Etten RL. Human liver acid phosphatases: purification and properties of a low molecular weight isoenzyme. Arch Biochem Biophys. 1982;214:505-15.

2. Ostrowski W, Barnard E. Evidence for a phosphoryl-enzyme intermediate in the catalytic reaction of prostatic acid phosphatase. Biochemistry. 1973;12:3893-7.
3. Chaimovich $\mathrm{H}$, Nome F. Purification and properties of three acid phosphatases from bovine brain. Arch Biochem Biophys. 1970;139:9-16.

4. Van Etten RL, Hickey ME. Phosphohistidine as a steichiometric intermediate in reactions catalized by isoenzymes in wheat germ acid phosphatase. Arch Biochem Biophys. 1977;183:250-9.

5. Andreu M, Alvarez EF, Lora Tamayo M. Investigación sobre fosfatasa. Purificación de la fosfatasa ácida de patata y caracterización de los grupos activos de la enzima. Anal Real Soc Español Fis-Quim. 1960;56:67-72.

6. Haak H, Guija E, Soberón M. Purificación y propiedades de la fosfatasa ácida de bajo peso molecular de hígado de cerdo. Bol Soc Quim Perú. 1993;59(3):149-57.

7. Boyer PD. The enzymes. Vol IV. 3rd ed. New York: Academic Press; 1970. p. 449-98.

8. Moss DW, Raymond FD, Wile DB. Clinical and biological aspects of acid phosphatase. Crit Rev Clin Lab Sci. 1995;82(4):431-67.

9. Lin MF, Li SS, Chu TM, Lee CL. Comparison of prostate acid phosphatase with acid phosphatase isoenzymes from the lung and spleen. J Clin Lab Anal. 1990;4(6):420-5.

10. Kamoshida S, Tsutsumi Y. Extraprostatic localization of prostatic acid phosphatase and prostate-specific antigen: distribution in cloacogenic glandular epithelium and sexdependent expression in human anal gland. Hum Pathol. 1990;21(11):1108-11.

11. Kishi Y, Kami M, Kusumi F, Mineyama T, Kato D, Hamaki $\mathrm{T}$, et al. Prostatic acid phosphatase (PAP): a possible diagnostic marker of intravascular large B-cell lymphoma. Haematol. 2004;89(4):e43-e45.

12. Haak H, Guija E, Soberón M. Mecanismo de acción de la fosfatasa ácida de hígado de bovino. Bol Soc Quim Perú. 1989;55(4):209-19.

13. Hickey EM, Waymack PP, Van Etten RL. pH-dependent leaving group effects on hydrolysis reactions of phosphate and phophonate esters catalyzed by wheat germ acid phosphatase. Arch Biochem Biophys. 1976;172:439-48.

14. Arauco F. Fosfatasa ácida de bajo peso molecular de hígado de alpaca. Purificación y caracterización. Tesis para obtener el grado de Magíster, Universidad Nacional Mayor de San Marcos; 1992.

15. Haak H, Guija E, Soberón M. Mecanismo de acción de la fosfatasa ácida de hígado de bovino. Bol Soc Quim Perú. 1983;55(4):209-19.

16. Nordlie R, Lardy H. Mamalian liver phosphoenolpyruvate carboxykinase activities. J Biol Chem. 1963;238:2259-63.

17. Lineweaver H,y Burk D. The determination of enzyme dissociation constants. J Am Chem Soc. 1934;56:658-66.

18. Petersford R, Beeson P. Fever of unexplained origin: report on 100 cases. Medicine (Baltimore). 1961;40:1-30.

19. Dikiy A, Funhoff E, Averill B, Ciurli S. New insights into the mechanism of purple acid phosphatase through ${ }^{1} \mathrm{H}$ NMR spectroscopy of the recombinant human enzyme. J Am Chem Soc. 2002;124(47):13974-5. 
20. Elliott T, Mitic N, Gahan L, Guddat L, Schenk G. Inhibition studies of purple acid phosphatases: implications of the catalytic mechanism. J Braz Chem Soc. 2006;17(8):155865.

21. Cleland WW. The kinetics of enzyme-catalyzed reactions with two or more substrates or products. Nomenclature and rate equations. Biochim Biophys Acta. 1963;67:104-37.

22. Guija E, Haak H, Soberón M. Fosfatasa ácida de hígado de cerdo. Inhibición por productos. Bol Soc Quim Perú. 1993;59(4):242-6.

23. Baldijao CE, Guija E, Bittencourt H, Chaimovich H. Steady state kinetics and effect of SH inhibitors on acid phosphatase from bovine brain. Biochim Biophys Acta. 1975;391:31625.

24. Haak H, Guija E. Efecto del glicerol sobre la catálisis por la fosfatasa ácida de hígado de bovino. Bol Soc Quim Perú. 1993;59(1):31-7.
25. Burdette R, Quinn D. Interfacial reaction dynamics and acylenzyme mechanism for lipoprotein lipase-catalyzed hydrolysis of lipid p-nitrophenyl esters. J Biol Chem. 1986;261:1201621.

Manuscrito recibido el 17 de octubre de 2007 y aceptado para publicación el 13 de diciembre de 2007.

\section{Correspondencia:}

Dr. Emilio Guija Poma

Centro de Investigación de Bioquímica y Nutrición

Facultad de Medicina, UNMSM

Av. Grau 755. Lima 1, Perú

Correo-e: eguijap@hotmail.com 